\title{
LIST OF COUNTRIES ADHERING TO THE UNION
}

The following is a list of the 46 countries that adhered to the Union in September 1970, giving also the year of admission, the approximate number of Members, and the Adhering Organizations.

\begin{tabular}{|c|c|c|c|}
\hline Country & Year & Members & Adhering Organizations \\
\hline Argentina & 1927 & 16 & Comité Nacional de Astronomía, La Plata. \\
\hline Australia & 1939 & 54 & $\begin{array}{l}\text { Australian National Committee for Astronomy, } \\
\text { Sydney. }\end{array}$ \\
\hline Austria & 1955 & 17 & Österreichische Akademie der Wissenschaften, Wien. \\
\hline Belgium & 1920 & 52 & $\begin{array}{l}\text { Académie Royale de Belgique, Bruxelles, Koninklijke } \\
\text { Vlaamse Academie van België, Brussel }\end{array}$ \\
\hline Brazil & 1961 & 10 & Conselho Nacional de Pesquisas, Rio de Janeiro. \\
\hline Bulgaria & 1957 & 9 & Bulgarian Academy of Sciences '7 November', Sofia. \\
\hline Canada & 1920 & 89 & $\begin{array}{l}\text { National Committee of Astronomy for Canada, Dept. } \\
\text { of Geophysics and Astronomy, University of British } \\
\text { Columbia, Vancouver 8, B.C. }\end{array}$ \\
\hline Chile & 1947 & 5 & Universidad de Chile, Santiago de Chile. \\
\hline Colombia & 1967 & 1 & $\begin{array}{l}\text { Academia Colombiana de Ciencias Exactas, Físicas y } \\
\text { Naturales, Bogotá. }\end{array}$ \\
\hline Cuba & 1970 & 0 & $\begin{array}{l}\text { Instituto de Astronomía de la Academia de Ciencias de } \\
\text { Cuba, La Habana }\end{array}$ \\
\hline Czechoslovakia & 1922 & 49 & $\begin{array}{l}\text { National Committee of Astronomy of Czechoslovakia, } \\
\text { Czechoslovak Academy of Sciences, Praha. }\end{array}$ \\
\hline Denmark & 1922 & 19 & $\begin{array}{l}\text { Danish National Committee for Astronomy, det } \\
\text { Kongelige Danske Videskabernes Selskab, København. }\end{array}$ \\
\hline Finland & 1948 & 10 & Finnish Academy of Sciences and Letters, Helsinki. \\
\hline France & 1920 & 168 & $\begin{array}{l}\text { Comité National Français d'Astronomie, Académie des } \\
\text { Sciences, Paris. }\end{array}$ \\
\hline F.R.G. & 1951 & 136 & Rat Westdeutscher Sternwarten zu München. \\
\hline G.D.R. & 1951 & 33 & $\begin{array}{l}\text { Nationalkomitee für Astronomie der DDR, Deutsche } \\
\text { Akademie der Wissenschaften zu Berlin. }\end{array}$ \\
\hline Greece & 1920 & 19 & $\begin{array}{l}\text { Greek National Committee for Astronomy, Academy } \\
\text { of Athens, Athens. }\end{array}$ \\
\hline Hungary & 1947 & 12 & Hungarian Academy of Sciences, Budapest. \\
\hline India & 1964 & 49 & $\begin{array}{l}\text { Indian National Committee for the International Astro- } \\
\text { nomical Union, Indian National Science Academy, } \\
\text { New Delhi. }\end{array}$ \\
\hline Iran & 1969 & $\mathbf{0}$ & Tehran University, Tehran. \\
\hline Ireland & 1947 & 6 & $\begin{array}{l}\text { National Committee of Astronomy, Royal Irish } \\
\text { Academy, Dublin. }\end{array}$ \\
\hline Israel & 1954 & 14 & $\begin{array}{l}\text { The Israel Academy of Sciences and Humanities, } \\
\text { Jerusalem. }\end{array}$ \\
\hline Italy & 1921 & 77 & Consiglio Nazionale delle Ricerche, Roma. \\
\hline Japan & 1920 & 105 & $\begin{array}{l}\text { National Committee of Astronomy of Japan, Science } \\
\text { Council of Japan, Tokyo. }\end{array}$ \\
\hline
\end{tabular}




\begin{tabular}{|c|c|c|c|}
\hline Country & Year & Members & Adhering Organizations \\
\hline Korea (PDR) & 1961 & 3 & Academy of Sciences, Phyongyang. \\
\hline Mexico & 1921 & 13 & Universidad Nacional de Mexico, Mexico D.F. \\
\hline Netherlands & 1922 & 64 & $\begin{array}{l}\text { Koninklijke Nederlandse Academie van Weten- } \\
\text { schappen, Amsterdam. }\end{array}$ \\
\hline New Zealand & 1964 & 5 & Royal Society of New Zealand, Wellington. \\
\hline Norway & 1922 & 14 & Det Norske Videnskaps-Akademi, Oslo. \\
\hline Poland & 1922 & 42 & Polska Akademia Nauk, Warsawa. \\
\hline Portugal & 1924 & 14 & $\begin{array}{l}\text { Seccao Portuguesa das Uniones Internacionais Astro- } \\
\text { nómica, Geodésica e Geofisica, Lisboa. }\end{array}$ \\
\hline Roumanie & 1928 & 18 & $\begin{array}{l}\text { Conseil Scientifique de l'Observatoire de Bucarest, } \\
\text { Bucarest. }\end{array}$ \\
\hline South Africa & 1938 & 13 & Council for Scientific and Industrial Research, Pretoria. \\
\hline Spain & 1922 & 17 & Comisión Nacional de Astronomía, Madrid. \\
\hline Sweden & 1925 & 42 & Kungl. Vetenskaps-Akademien, Stockholm. \\
\hline Switzerland & 1923 & 21 & Schweizerische Naturforschende Gesellschaft, Basel. \\
\hline Taiwan (Republic & & & \\
\hline $\begin{array}{l}\text { of China) } \\
\text { Turkey }\end{array}$ & $\begin{array}{l}1959 \\
1961\end{array}$ & $\begin{array}{r}9 \\
18\end{array}$ & $\begin{array}{l}\text { Academia Sinica, Nankang, Taipei. } \\
\text { Türk Astronomi Dernegi, Istanbul. }\end{array}$ \\
\hline U.A.R. & 1925 & 9 & Astronomical Center at Helwan, Egyptian Region. \\
\hline United Kingdom & 1920 & 237 & The Royal Society, London. \\
\hline Uruguay & 1970 & 0 & $\begin{array}{l}\text { Universidad de la República, Comité Nacional de } \\
\text { Astronomía, Montevideo. }\end{array}$ \\
\hline U.S.A. & 1920 & 728 & The National Academy of Sciences, Washington D.C. \\
\hline U.S.S.R. & 1920 & 362 & Academy of Sciences of the U.S.S.R., Moscow. \\
\hline Vatican City State & 1932 & 7 & Pontificia Academia delle Scienze, Città del Vaticano. \\
\hline Venezuela & 1953 & 2 & $\begin{array}{l}\text { Academia de Ciencias Físicas, Matemáticas y Natura- } \\
\text { les, Caracas. }\end{array}$ \\
\hline Yugoslavia & 1935 & 14 & $\begin{array}{l}\text { Conseil Fédéral pour la Coordination de la Recherche } \\
\text { Scientifique, Belgrade. }\end{array}$ \\
\hline
\end{tabular}

